\title{
Experimental Realization of Strong Effective Magnetic Fields in an Optical Lattice
}

\author{
M. Aidelsburger, ${ }^{1,2}$ M. Atala, ${ }^{1,2}$ S. Nascimbène, ${ }^{1,2,3}$ S. Trotzky, ${ }^{1,2}$ Y.-A. Chen, ${ }^{1,2, *}$ and I. Bloch ${ }^{1,2, \dagger}$ \\ ${ }^{1}$ Fakultät für Physik, Ludwig-Maximilians-Universität, Schellingstrasse 4, 80799 München, Germany \\ ${ }^{2}$ Max-Planck-Institut für Quantenoptik, Hans-Kopfermann-Strasse 1, 85748 Garching, Germany \\ ${ }^{3}$ Laboratoire Kastler Brossel, CNRS, UPMC, Ecole Normale Supérieure, 24 rue Lhomond, 75005 Paris, France
}

(Received 26 September 2011; published 12 December 2011)

\begin{abstract}
We use Raman-assisted tunneling in an optical superlattice to generate large tunable effective magnetic fields for ultracold atoms. When hopping in the lattice, the accumulated phase shift by an atom is equivalent to the Aharonov-Bohm phase of a charged particle exposed to a staggered magnetic field of large magnitude, on the order of 1 flux quantum per plaquette. We study the ground state of this system and observe that the frustration induced by the magnetic field can lead to a degenerate ground state for noninteracting particles. We provide a measurement of the local phase acquired from Raman-induced tunneling, demonstrating time-reversal symmetry breaking of the underlying Hamiltonian. Furthermore, the quantum cyclotron orbit of single atoms in the lattice exposed to the magnetic field is directly revealed.
\end{abstract}

DOI: 10.1103/PhysRevLett.107.255301

The application of strong magnetic fields to twodimensional electron gases has led to the discovery of seminal quantum many-body phenomena, such as the integer and fractional quantum Hall effect [1]. Ultracold atoms constitute a unique experimental system for studying such systems in a clean and well-controlled environment and for exploring new physical regimes, not attainable in typical condensed matter systems [2,3]. However, charge neutrality of atoms prevents direct application of the Lorentz force with a magnetic field. An equivalent effect can be provided by the Coriolis force in a rotating atomic gas, which led to the observation of quantized vortices in a Bose-Einstein condensate [4]. The regime of fast rotation, in which the atomic gas occupies the lowest Landau level, was achieved in Refs. [5] but the amplitude of the effective gauge field remained too small to enter the strongly correlated regime that requires a number of vortices on the order of the particle number $[2,6]$. An alternative route consists in applying Raman lasers to the gas in order to realize a Berry's phase for a moving particle $[7,8]$. The effective gauge fields generated in such a setup resulted in the observation of a few vortices, but were still far from the strong-field regime.

In this Letter, we demonstrate the creation of strong effective magnetic fields for ultracold atoms in a twodimensional optical lattice. Inspired by the proposal of Jaksch and Zoller [9] and subsequent work [10-12], our technique is based on atom tunneling assisted by Raman transitions [see Fig. 1(a)]. Because of the spatial variation of the Raman coupling, the wave function of an atom tunneling from one lattice site to another acquires a nontrivial phase, which can be interpreted as an effective Aharonov-Bohm phase. In our setup, the magnetic flux per four-site plaquette is staggered with a zero mean, alternating between $\pi / 2$ and $-\pi / 2$ [see Fig. 1(b)] [13]. We study the nature of the ground state in this optical
PACS numbers: 67.85. $-\mathrm{d}$, 03.65.Vf, 03.75.Lm, 73.20. $-\mathrm{r}$

lattice from its momentum distribution and show, in particular, that the frustration associated with the effective magnetic field can lead to a degenerate ground state for single particles, similar to the prediction of Ref. [14]. We also study the quantum cyclotron dynamics of single atoms restricted to a four-site plaquette and obtain direct evidence for time-reversal symmetry breaking of the Hamiltonian.

Our experimental setup consists of an ultracold gas of ${ }^{87} \mathrm{Rb}$ atoms held in a two-dimensional square lattice, forming an array of 1D Bose gases. The lattice was created by two standing waves of laser light at $\lambda_{s}=767 \mathrm{~nm}$ ("short" lattices) and a third one with twice the wavelength

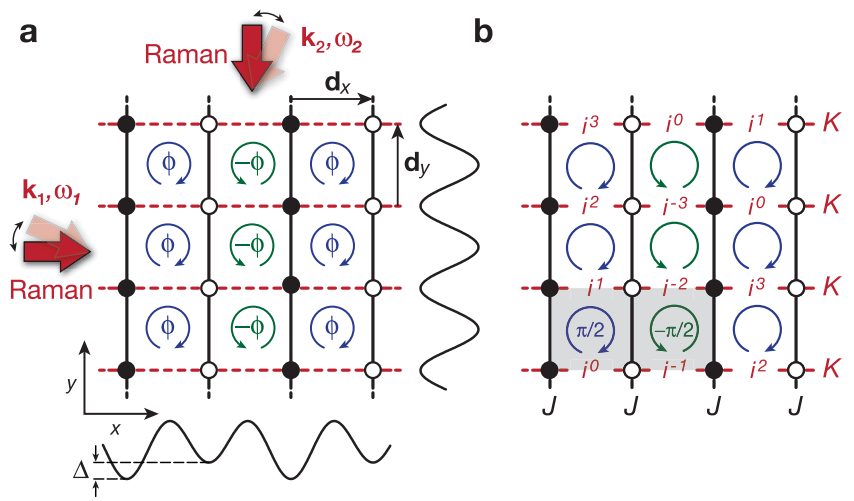

FIG. 1 (color). Experimental setup. (a) The experiment consists of a $2 \mathrm{D}$ array of $1 \mathrm{D}$ potential tubes with spacing $\left|\mathbf{d}_{x}\right|=$ $\left|\mathbf{d}_{y}\right|=\lambda_{s} / 2$. While bare tunneling occurs along the $y$ direction with amplitude $J$, it is inhibited along $x$ owing to a staggered potential offset $\Delta$. A pair of Raman lasers with wave vectors $\mathbf{k}_{1,2}$ and frequency difference $\omega_{1}-\omega_{2}=\Delta / \hbar$, induces a resonant tunnel coupling of magnitude $K$ whose phase depends on position. This realizes an effective flux $\pm \phi$ per plaquette with alternating sign along $x$. (b) Spatial distribution of the phase of the Raman-induced tunnel coupling realized in the experiment. The gray shaded area highlights the magnetic unit cell. 
("long" lattice, $\lambda_{l} \simeq 2 \lambda_{s}$ ) to generate a staggered potential with amplitude $\Delta$ as shown in Fig. 1(a). A pair of Raman lasers then induced tunneling along the staggered direction. Let us consider the Raman-assisted tunneling of an atom from a site of low energy at $\mathbf{R}=m \mathbf{d}_{x}+n \mathbf{d}_{y}$ to a site of high energy at $\mathbf{R}+\mathbf{d}_{x}$. Assuming $\omega_{1}>\omega_{2}$, one obtains $K(\mathbf{R})=K e^{-i \delta \mathbf{k} \cdot \mathbf{R}}$, where $\delta \mathbf{k}=2 \pi / \lambda_{K}\left(\mathbf{e}_{\mathbf{k}_{1}}-\mathbf{e}_{\mathbf{k}_{2}}\right)$ denotes the wave vector [15]. The system is then effectively described by the noninteracting Hamiltonian

$$
\hat{H}=-\sum_{\mathbf{R}}\left(K e^{ \pm i \delta \mathbf{k} \cdot \mathbf{R}} \hat{a}_{\mathbf{R}}^{\dagger} \hat{a}_{\mathbf{R}+\mathbf{d}_{x}}+J \hat{a}_{\mathbf{R}}^{\dagger} \hat{a}_{\mathbf{R}+\mathbf{d}_{y}}\right)+\text { H.c. }
$$

where the sign of the phase factor is positive for even sites of $x$ and negative otherwise.

The phase factors in $K(\mathbf{R})$ can be interpreted as Aharonov-Bohm phases. For the propagation of the Raman beams shown in Fig. 1(a) along $x$ and $-y$ and $\lambda_{K}=\lambda_{l}$, we obtain a phase factor of $\delta \mathbf{k} \cdot \mathbf{R}=\frac{\pi}{2}(m+n)$. Therefore the phase accumulated on a closed path around a plaquette is equal to $\phi=\pi / 2$, alternating in sign along the $x$ direction. A different value of the flux $\phi$ could be achieved by choosing a different wavelength for the Raman lasers or by using a different angle between them, allowing for a fully tunable flux per plaquette in our setup.

Our experiment started by loading a Bose-Einstein condensate of about $5 \times 10^{4}$ atoms into a staggered $2 \mathrm{D}$ optical lattice as shown in Fig. 1(a) with $\Delta / h=4.4(1) \mathrm{kHz}$, resulting in an array of tubes with no coherence along $x$ [15]. We then switched on the Raman lasers on resonance with strength $V_{K}^{0}=0.49(1) E_{r}$ to restore the coherence. In the limit $V_{K}^{0} \ll \Delta$, the amplitude of Raman-induced tunnel coupling is $K \simeq J_{x} V_{K}^{0} /(2 \sqrt{2} \Delta)$, with $J_{x}$ being the bare tunnel coupling along $x$. For our experimental parameters, this yields a value of $K=2 \pi \times 59(2) \mathrm{Hz}$, in agreement with an independent measurement of $K=2 \pi \times 61(3) \mathrm{Hz}$ [15]. After holding the atoms in this configuration for $10 \mathrm{~ms}$, we observed a momentum distribution with restored phase coherence as shown in Fig. 2(b). This can be attributed to a redistribution of entropy present in the random phases between the 1D condensates into their longitudinal modes.

To understand the momentum distribution, we calculated the band structure of this lattice in the tight-binding approximation according to Ref. [14]. In the presence of the gauge field, the Hamiltonian remains periodic. However, the magnetic unit cell contains two nonequivalent sites, leading to a splitting of the tight-binding band structure into two subbands [15-17]. The frustration introduced by the position-dependent phase factors in $K(\mathbf{R})$ causes the phase of the atomic wave function to be nonuniform, leading to a condensation at nonzero momenta. In the case $J / K=1$, we obtain a nondegenerate ground state. However, the wave function itself consists of two momentum components; therefore, we observe two diffraction peaks within the first magnetic Brillouin zone, one being shifted by $\Delta \mathbf{k}=\left(k_{s} / 4, k_{s} / 4\right)$ with respect to the minimum

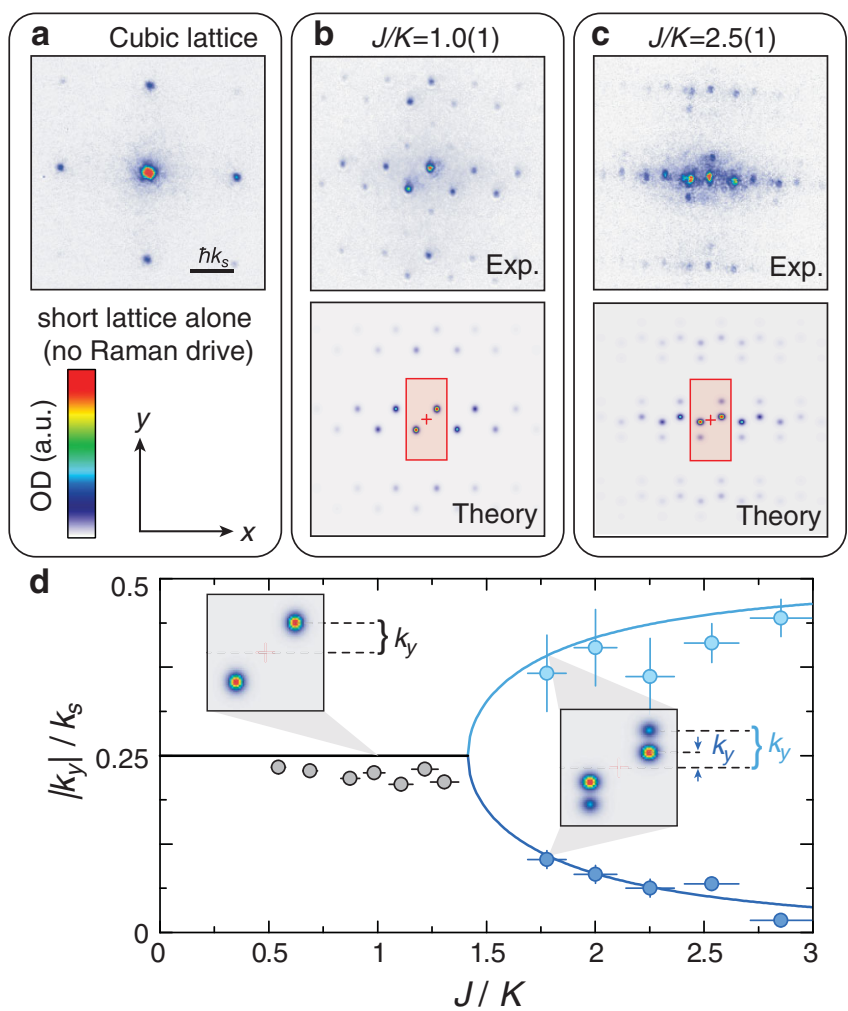

FIG. 2 (color). Momentum distribution measured after a time of flight of $20 \mathrm{~ms}$ for (a) simple cubic lattice, (b) $J / K=1.0(1)$ and (c) $J / K=2.5(1)$. The latter two are compared with theoretical profiles obtained by an exact diagonalization of Hamiltonian (1) on a $31 \times 31$ lattice with harmonic confinement [15]. Red squares in the theoretical profiles indicate the magnetic Brillouin zone and the crosses mark the center. (d) Projection along $y$ of the momentum peaks located at $k_{x}=+k_{s} / 4$, as a function of $J / K$. For $J / K<\sqrt{2}$ the peaks are located at $k_{y}=$ $k_{s} / 4$, while for $J / K>\sqrt{2}$ the peaks are split due to the emergent ground-state degeneracy (see insets). The solid lines correspond to the minima of the lowest Bloch band for a translationally invariant system [15].

of the dispersion relation [15]. Because of the unit cell containing more than one lattice site, the size of the magnetic Brillouin zone is reduced compared to the one of the square lattice [see Fig. 3(a)]. Therefore the ground-state momentum distribution exhibits several peaks in the shortlattice Brillouin zone, whose measured positions are in good agreement with the quasimomenta of the Bloch states of lowest energy [see Fig. 2(b)].

When varying the ratio $J / K$ by adjusting the $y$-lattice depth, we observed the positions and number of peaks in the momentum distribution to remain unchanged for $J / K \lesssim 1.4$ [see Fig. 2(d)]. Above this value, additional peaks appear [see Fig. 2(c)], which correspond to the population of two degenerate ground states. This behavior agrees with the band-structure calculation, which shows a bifurcation at $J / K=\sqrt{2}$, above which the energy minimum is split into two degenerate ground states [see 

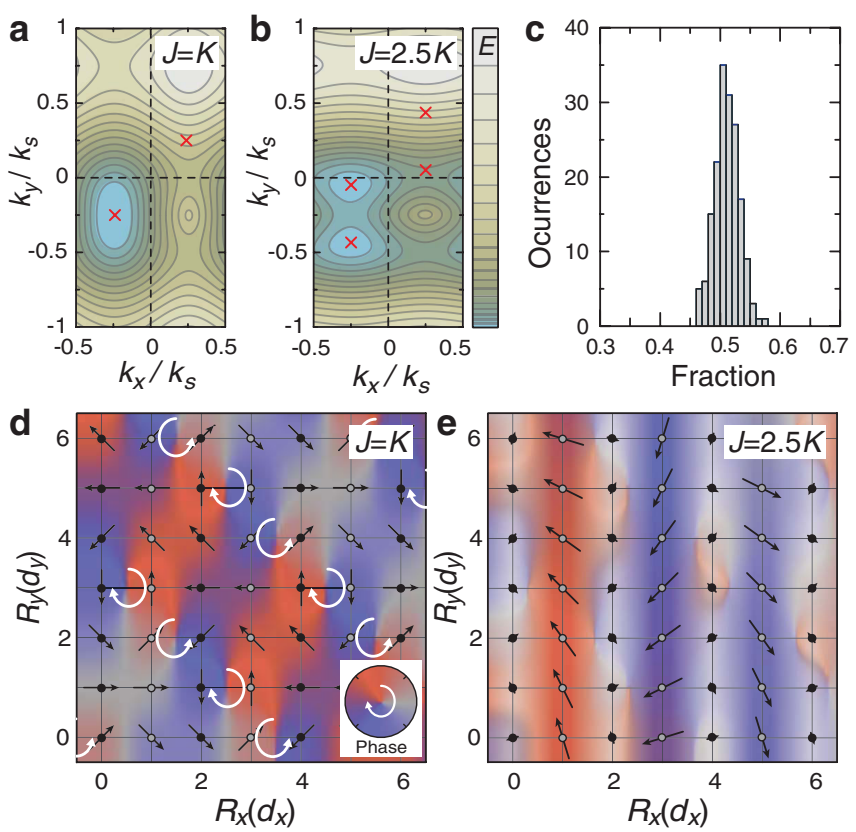

FIG. 3 (color). Dispersion relation of the lowest Bloch band, calculated in the tight-binding approximation [15] for (a) $J / K=1$ and (b) $J / K=2.5$ and plotted for the first magnetic Brillouin zone. The red crosses mark the positions of the corresponding momentum peaks together with the ones shifted due to the intrinsic structure of the wave function. (c) Histogram of the measured fraction of atoms in peaks corresponding to the lower momentum state for $J / K=2.5$. The measurement was performed over 172 identical experimental runs. (d)-(e) Spatial distribution of the phase and atomic density (color brightness) for the ground-state wave function [15]. The vortices with different chirality in the phase distribution for $J / K=1$ (d) are illustrated by the rotation of the white arrows. While in this case the atomic density is uniform, it exhibits a charge density wave for $J / K=2.5$ (e). For the second degenerate ground state we observe similar behavior but the density pattern is shifted by one lattice site.

Fig. 3(b)]. We find the measured and predicted peak positions of these two ground states to be in good agreement [see Fig. 2(d)]. The nature of the bifurcation is identical to the one predicted in Ref. [14] where it is induced by a variation of the magnetic flux amplitude at a fixed value of $J / K=1$. As shown in Fig. 3(d), the atomic density is uniform for $J / K=1$, while in the case $J / K=2.5$ it is strongly modulated for both single-particle ground states [see Fig. 3(e)]. For $J>\sqrt{2} K$ the bare coupling dominates and the phases of the atomic wave function tend to align along the $y$ direction, thereby frustrating the phase relation imposed by the Hamiltonian. As a consequence, the density in every second stripe along $y$ is suppressed. Contrary to the case of a triangular lattice with frustrated hopping studied in [18], the atom fraction in each single-particle ground state does not fluctuate, as shown in Fig. 3(c), and we observe an equal population in both states as predicted for interacting [14] or finite size systems [19].
In order to directly reveal time-reversal symmetry breaking of the Hamiltonian, we probed the local structure of the lattice with the artificial gauge field at the level of a foursite square plaquette, which allows us to isolate plaquettes with equal sign of the flux. This was achieved by applying superlattice potentials along both the $x$ and $y$ directions, and in order to avoid coupling to axial modes along the potential tubes, an additional lattice along the $z$ direction $\left(\lambda_{z}=844 \mathrm{~nm}\right)$ was used in these measurements. The four sites of a single plaquette are denoted as $A, B, C, D$ [see inset Fig. 4(a)]. The relative phases of the long and
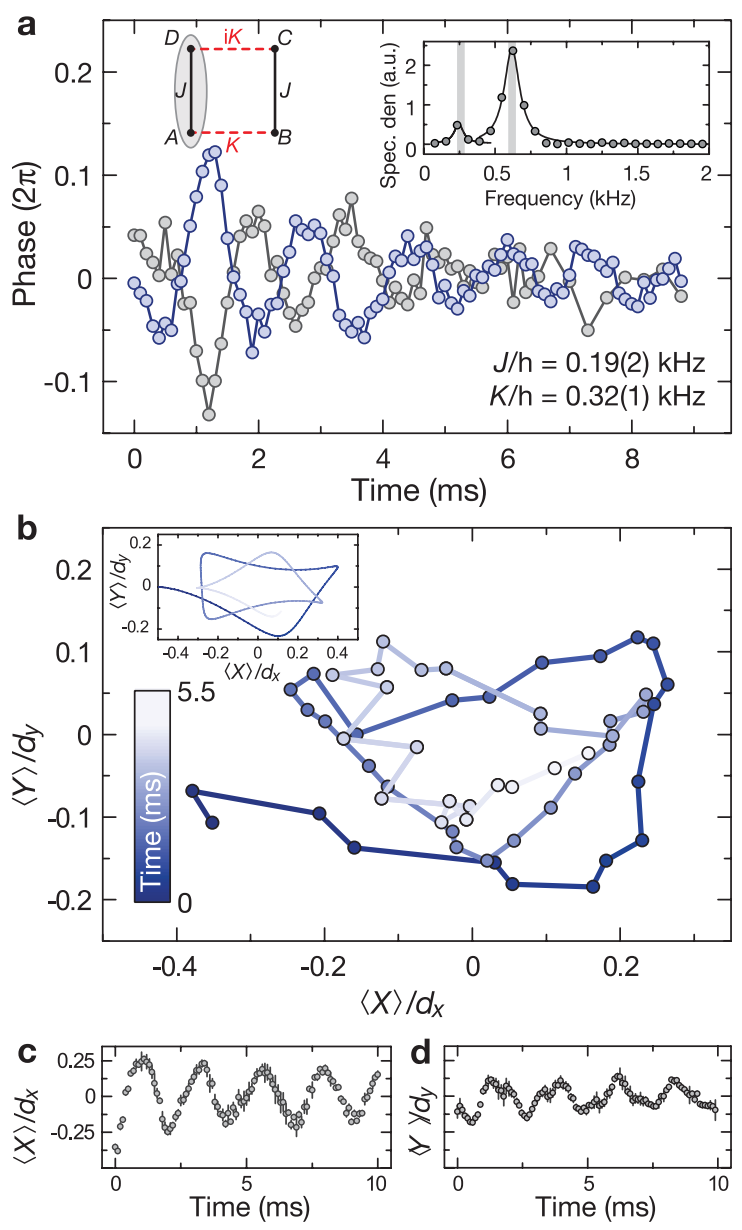

FIG. 4 (color). Time-reversal symmetry breaking and cyclotron orbits. (a) Phase evolution of the double-slit pattern along $y$ (integrated along $x$ ), as a function of time for $\hbar \omega=\Delta$ (blue) and $\hbar \omega=-\Delta$ (gray). The inset shows the Fourier transformation for $\hbar \omega=\Delta$ depicting two frequency components at $0.24(6) \mathrm{kHz}$ and $0.62(13) \mathrm{kHz}$, in good agreement with theory (vertical lines). (b) Cyclotron orbits of the average particle position obtained from the mean atom positions $\langle X\rangle / d_{x}$ (c) and $\langle Y\rangle / d_{y}$ (d) for $J / h=0.50(2) \mathrm{kHz}, \quad K / h=0.27(1) \mathrm{kHz}, \quad$ and $\Delta / h=$ $5.05(2) \mathrm{kHz}$. Each data point is an average over three measurements. The inset in (b) shows the theoretical curve calculated for $\phi=0.80 \times \pi / 2$ and a $1 / e$-damping time of $13 \mathrm{~ms}$ obtained from damped sine fits to $\langle X\rangle / d_{x}$ and $\langle Y\rangle / d_{y}$. 
short lattices were adjusted so that the plaquettes were symmetric along $y$ and tilted along $x$, with an energy offset $\Delta / h=6.0(1) \mathrm{kHz}$. We first loaded single atoms in the ground state of the tilted plaquettes $\left|\psi_{1}\right\rangle=(|A\rangle+$ $|D\rangle) / \sqrt{2}$, and subsequently switched on the Raman lasers with $\hbar \omega=\Delta$ in order to induce resonant coupling to the $B$ and $C$ sites. In the limit $J \ll K$, the dynamics along $y$ would be suppressed and the initial state $\left|\psi_{1}\right\rangle$ couples to the state $\left|\psi_{2}\right\rangle=(|B\rangle+i|C\rangle) / \sqrt{2}$, where the relative phase is induced by the Raman lasers. In our case $(J / K \approx 0.5)$ the evolution of the imprinted phase factors is more complex. We measured this evolution through the shape of the momentum distribution obtained after time of flight [15]. This dynamics is a direct consequence of the complex phase factor, revealing the time-reversal symmetry breaking of the Hamiltonian [see Fig. 4(a)]. For $\omega=-\Delta / \hbar$ the role of the Raman beams is exchanged leading to a sign reversal of the phase evolution.

We also investigated this dynamics in real space in order to exhibit the influence of the gauge field on the particle flow. By generalizing the site-resolved measurement technique performed in [20] for an array of double wells to plaquettes, we measured the atom population per site $N_{q}$ ( $q=A, B, C, D)$ [15], thus obtaining the average atom positions $\langle X\rangle=\left(-N_{A}+N_{B}+N_{C}-N_{D}\right) d_{x} / 2 N \quad$ and $\langle Y\rangle=\left(-N_{A}-N_{B}+N_{C}+N_{D}\right) d_{y} / 2 N$, with $N$ being the total atom number. In the initial state $\left|\psi_{1}\right\rangle$ the atoms occupy the left wells $A$ and $D$ with equal weights. After switching on the Raman lasers we observe a coherent particle flow inside the plaquettes. Besides the particle current towards the right wells $B$ and $C$ [see Fig. 4(c)], we observe a deviation of the mean atom position along $y$ [see Fig. 4(d)]. This behavior is reminiscent of the Lorentz force acting on a charged particle in a magnetic field. As shown in Fig. 4(b), the mean atom position follows an orbit that is a small-scale quantum analog of the classical cyclotron orbits for charged particles. This coherent evolution is damped due to spatial inhomogeneities in the atomic sample. Having independently calibrated the values of $J$ and $K$, we fit from the measured atom dynamics the value of the magnetic flux $\phi=0.73(5) \times \pi / 2$. The difference from the value $\phi=\pi / 2$ expected for a homogeneous lattice stems from the smaller distance between lattice sites inside the plaquettes when separated. For the parameters used in Figs. 4(b)-4(d)] we calculate a distance $d_{y}=$ $0.78(1) \times \lambda_{s} / 2$ yielding $\phi=0.80(1) \times \pi / 2$, which qualitatively explains the measured flux value. Residual deviations might be due to an angle mismatch between the Raman beams and the lattices beams.

In conclusion, we have demonstrated a new type of optical lattice that realizes strong effective magnetic fields and breaks time-reversal symmetry. We have shown that the atomic sample relaxes to the minima of the magnetic band structure, realizing an analogue of a frustrated classical spin system. However, the spatial average of the magnetic flux is zero; hence, the Bloch band is topologically trivial $[10,17,21]$. By using a superlattice potential with more than two nonequivalent sites [10] or a linear tilt potential [9], it is possible to create a lattice with a uniform and nonzero magnetic flux. This system would realize the Harper Hamiltonian [22] and lead to the fractal band structure of the Hofstadter butterfly [23]. In particular the lowest band would exhibit a Chern number of one and be analogous to the lowest Landau level $[8,9,12,24]$. Our work constitutes an important step towards the study of quantum Hall effect with ultracold atomic gases and the creation of strongly interacting liquids such as the Laughlin state [25].

We acknowledge insightful discussions with $\mathrm{N}$. Cooper. We also thank S. Fölling for his help in illustrating our data. This work was supported by the DFG (FOR635, FOR801), the EU (STREP, NAMEQUAM, Marie Curie Fellowship to S. N.), and DARPA (OLE program).

*yu-ao.chen@1mu.de immanuel.bloch@mpq.mpg.de

[1] D. Tsui, H. Stormer, and A. Gossard, Phys. Rev. Lett. 48, 1559 (1982); R. Laughlin, ibid. 50, 1395 (1983).

[2] I. Bloch, J. Dalibard, and W. Zwerger, Rev. Mod. Phys. 80, 885 (2008).

[3] A. Fetter, Rev. Mod. Phys. 81, 647 (2009); N. Cooper, Adv. Phys. 57, 539 (2008).

[4] K. Madison, F. Chevy, W. Wohlleben, and J. Dalibard, Phys. Rev. Lett. 84, 806 (2000); J. Abo-Shaeer, C. Raman, J. Vogels, and W. Ketterle, Science 292, 476 (2001).

[5] V. Schweikhard et al., Phys. Rev. Lett. 92, 40404 (2004); V. Bretin, S. Stock, Y. Seurin, and J. Dalibard, ibid. 92, 50403 (2004).

[6] N. Cooper, N. Wilkin, and J. Gunn, Phys. Rev. Lett. 87, 120405 (2001).

[7] Y. Lin et al., Nature (London) 462, 628 (2009).

[8] J. Dalibard, F. Gerbier, G. Juzeliūnas, and P. Öhberg, arXiv:1008.5378 [Reviews of Modern Physics (Colloquium) (to be published)].

[9] D. Jaksch and P. Zoller, New J. Phys. 5, 56 (2003).

[10] F. Gerbier and J. Dalibard, New J. Phys. 12, 033007 (2010).

[11] A. Kolovsky, Europhys. Lett. 93, 20003 (2011).

[12] E. Mueller, Phys. Rev. A 70, 041603 (2004).

[13] L. Lim, C. Smith, and A. Hemmerich, Phys. Rev. Lett. 100, 130402 (2008); L. Lim, A. Hemmerich, and C. Smith, Phys. Rev. A 81, 023404 (2010).

[14] G. Möller and N. Cooper, Phys. Rev. A 82, 063625 (2010).

[15] See Supplemental Material at http://link.aps.org/ supplemental/10.1103/PhysRevLett.107.255301 for the band structure calculation, the experimental sequence, the Raman-assisted tunneling, and the detection and data analysis methods.

[16] E. Blount, Phys. Rev. 126, 1636 (1962).

[17] Y. Wang and C. Gong, Phys. Rev. B 74, 193301 (2006).

[18] J. Struck et al., Science 333, 996 (2011). 
[19] In trapped systems the degeneracy is slightly lifted and the two lowest energy states are symmetric and antisymmetric superpositions of the two states.

[20] S. Fölling et al., Nature (London) 448, 1029 (2007).

[21] F. Haldane, Phys. Rev. Lett. 61, 2015 (1988).

[22] P. Harper, Proc. Phys. Soc. London Sect. A 68, 874 (1955).

[23] D. Hofstadter, Phys. Rev. B 14, 2239 (1976).
[24] N. Cooper, Phys. Rev. Lett. 106, 175301 (2011); N. Cooper and J. Dalibard, Europhys. Lett. 95, 66004 (2011).

[25] A. Sørensen, E. Demler, and M. Lukin, Phys. Rev. Lett. 94, 86803 (2005); R. Palmer and D. Jaksch, ibid. 96, 180407 (2006); M. Hafezi, A. Sørensen, E. Demler, and M. Lukin, Phys. Rev. A 76, 023613 (2007). 